\title{
Double-averaged velocity and stress distributions for hydraulically-smooth and transitionally-rough turbulent flows
}

\author{
Stuart M. CAMERON ${ }^{1}$, Vladimir I. NIKORA ${ }^{1}$, \\ and Stephen E. COLEMAN ${ }^{2}$ \\ ${ }^{1}$ School of Engineering, University of Aberdeen, Aberdeen, United Kingdom \\ e-mails: s.cameron@abdn.ac.uk (corresponding author); v.nikora@abdn.ac.uk \\ ${ }^{2}$ Department of Civil and Environmental Engineering, The University of Auckland, \\ Auckland, New Zealand; e-mail: s.coleman@auckland.ac.nz
}

\begin{abstract}
We analyse experimental measurements of turbulent open-channel flow over hydraulically-smooth and transitionally-rough beds using the double-averaging methodology. Oil with a viscosity of $15 \times 10^{-6} \mathrm{~m}^{2} / \mathrm{s}$ is used instead of water so that transitional-range roughness Reynolds numbers can be achieved with large $(11.1 \mathrm{~mm})$ roughness elements, allowing spatial variations in the mean velocity field to more easily be measured. Distributions of double-averaged velocities, turbulence intensities, form-induced intensities, and viscous, Reynolds, form-induced and total shear stresses are studied with comparisons made between distributions for hydraulically-smooth, transitionally-rough, and fully-rough boundaries. Measured streamwise turbulence intensities for all experiments peaked at a constant distance from the bed $\left(z^{+}+d^{+}=15\right)$ when elevation scale is adjusted using the zero-plane displacement $d$ for the logarithmic velocity distribution. This collapse suggests that turbulence intensity distributions may be useful in assessing appropriate values of $d$ for transitionally-rough and fully-rough boundaries. Form-induced normal and shear stresses above the roughness tops were found to collapse towards a common curve independent of roughness Reynolds number.
\end{abstract}

Key words: double-averaging methodology, open-channel flow, particle image velocimetry.

(C) 2008 Institute of Geophysics, Polish Academy of Sciences 


\section{INTRODUCTION}

Bed roughness for turbulent channel flows can be classified into three regimes: (i) hydraulically-smooth - where surface roughness elements are completely submerged within the viscous sublayer and have little influence on the flow, (ii) transitionally-rough - where roughness elements are only partially submerged within the viscous sublayer and both roughness and viscous effects are important, and (iii) fully-rough - where roughness elements disrupt the viscous and buffer layers and velocity distributions become independent of viscosity. Hydraulically-smooth boundaries are suggested to exist for roughness Reynolds numbers $R_{k^{*}}<4$, while fullyrough boundaries develop when $R_{k^{*}}>60$ (e.g., Monin and Yaglom 1971), where $R_{k^{*}}=u_{*} k / v, u *$ is the shear velocity, $k$ is the roughness height, and $v$ is the kinematic fluid viscosity. Extensive discussion on the features of hydraulically-smooth, transitionally-rough and fully-rough boundary flows can be found in Grass (1971), Krogstad et al. (1992), Ligrani and Moffat (1986), and Schultz and Flack (2007).

The Reynolds-averaged (time or ensemble) Navier-Stokes equations are often used for interpreting measured or simulated velocity field data. Their application to transitionally-rough and fully-rough bed flows, however, leads to definitions of mean velocities and fluid stresses that do not account for the spatially-heterogeneous nature of these flows. The double-averaging (in time and in space) framework leads to improved definitions of fluid velocities and stresses by explicitly accounting for spatial variability. For steady, uniform, two-dimensional flow above roughness tops, the double-averaged momentum equations provide a new definition for total fluid shear stress $\langle\bar{\tau}\rangle$ in the primary flow direction (Nikora et al. 2007a):

$$
\langle\bar{\tau}\rangle=-\rho\langle\tilde{u} \tilde{w}\rangle-\rho\left\langle\overline{u^{\prime} w^{\prime}}\right\rangle+\mu \frac{\mathrm{d}\langle\bar{u}\rangle}{\mathrm{d} z},
$$

where the terms on the right hand side of (1) are form-induced, Reynolds and viscous shear stresses, respectively, $u$ and $w$ are the streamwise and bednormal velocities, angle brackets and straight overbars indicate spatial and time averaging, respectively, the prime and wavy overline represent time and spatial fluctuations, $z$ is the elevation with respect to the roughness tops, $\mu=\rho v$ is the dynamic fluid viscosity, and $\rho$ is the fluid density. We can also define form-induced normal stresses as $-\rho\langle\tilde{u} \tilde{u}\rangle$ and $-\rho\langle\tilde{w} \widetilde{w}\rangle$, and

spatially-averaged Reynolds normal stresses as $-\rho\left\langle\overline{u^{\prime} u^{\prime}}\right\rangle$ and $-\rho\left\langle\overline{w^{\prime} w^{\prime}}\right\rangle$.

Some experimentally measured distributions of these double-averaged quantities are available for fully-rough bed flows where viscous effects can generally be neglected (e.g., Nikora et al. 2007b), but no measurements are yet available for transitionally-rough boundaries where viscous stresses are 
not negligible and the potential significance of form-induced stresses is unknown. Within this paper, we focus on transitionally-rough-bed flows and present experimental measurements of double-averaged fluid velocities and stresses with comparisons made to measured distributions for smooth bed flows.

\section{EXPERIMENTS}

Experiments were conducted in a $0.40 \mathrm{~m}$ wide, $10.0 \mathrm{~m}$ long, glass-sided tilting flume at the University of Auckland, New Zealand. The flume was specially designed to circulate oil with a viscosity higher than that of water, enabling low Reynolds number turbulent flows with increased viscous sublayer thickness to be studied. The oil used was Shell Ondina 15, a transparent mineral oil with a kinematic viscosity of $v=15 \times 10^{-6} \mathrm{~m}^{2} / \mathrm{s}$ and a density of $\rho=850 \mathrm{~kg} / \mathrm{m}^{3}$ at $40^{\circ} \mathrm{C}$. Heating elements connected to a thermostat in the flume exit tank raised the oil to $40^{\circ}$ and maintained a constant temperature throughout the experiments. The flow was driven by a positive displacement pump with a maximum flow rate of $80 \mathrm{l} / \mathrm{s}$.

Two sets of experiments were carried out: ' $\mathrm{M}$ ' series with $11.1 \mathrm{~mm}$ diameter $D$ glass marbles covering the bed, and ' $\mathrm{S}$ ' series with a hydraulically-smooth bed surface (Table 1). For the ' $M$ ' series experiments glass

Table 1

Experimental parameters: $U$ is the depth averaged velocity, $H$ is the flow depth above the roughness tops, $S_{0}$ is the bed surface slope, $v$ is the kinematic fluid viscosity, $D$ is the particle diameter, $u_{*}$ is the shear velocity calculated from the total fluid stress at the roughness tops, $R e=U H / v$ is the bulk Reynolds number, $R_{k^{*}}=u * k / v$ is the roughness Reynolds number, and $F r=U /(g H)^{0.5}$ is the Froude number.

\begin{tabular}{|c|c|c|c|c|c|c|c|c|c|}
\hline & $\begin{array}{c}U \\
{[\mathrm{~m} / \mathrm{s}]}\end{array}$ & $\begin{array}{c}H \\
{[\mathrm{~mm}]}\end{array}$ & $S_{0}$ & $\begin{array}{c}v \\
{\left[\times 10^{-6} \mathrm{~m}^{2} / \mathrm{s}\right]}\end{array}$ & $\begin{array}{c}D \\
{[\mathrm{~mm}]}\end{array}$ & $\begin{array}{c}u_{*} \\
{[\mathrm{~m} / \mathrm{s}]}\end{array}$ & $R e$ & $R_{k^{*}}$ & $F r$ \\
\hline M1 & 0.468 & 98.0 & 0.0012 & 15.1 & 11.1 & 0.031 & 3114 & 11.4 & 0.48 \\
M2 & 0.554 & 98.5 & 0.0016 & 15.0 & 11.1 & 0.036 & 3720 & 13.3 & 0.56 \\
M3 & 0.631 & 98.5 & 0.0023 & 15.0 & 11.1 & 0.041 & 4237 & 15.2 & 0.64 \\
M4 & 0.718 & 99.0 & 0.0028 & 15.0 & 11.1 & 0.047 & 4846 & 17.4 & 0.73 \\
M5 & 0.778 & 100.0 & 0.0033 & 15.1 & 11.1 & 0.052 & 5281 & 19.1 & 0.79 \\
S1 & 0.480 & 98.5 & 0.0009 & 15.0 & smooth & 0.030 & 3223 & $<<1$ & 0.49 \\
S2 & 0.645 & 98.5 & 0.0014 & 15.1 & smooth & 0.038 & 4314 & $<<1$ & 0.66 \\
S3 & 0.830 & 98.0 & 0.0024 & 15.0 & smooth & 0.047 & 5546 & $<<1$ & 0.85 \\
\hline
\end{tabular}


spheres were glued in a tightly-packed hexagonal arrangement 1 layer deep over most of the flume and deepened to 3 layers across a $1.0 \mathrm{~m}$ long test section located $7.0 \mathrm{~m}$ from the flume entrance. The studied flows had fullyturbulent range Reynolds numbers $R e=U H / v$ between 3114 and 5546, and subcritical range Froude numbers $F r=U /(g H)^{0.5}$ to a maximum of 0.85 (where $U$ is the depth-averaged velocity, $H$ is the flow depth above the roughness tops, and $g$ is the acceleration due to gravity). Roughness Reynolds numbers $R_{k^{*}}=k u * / v$ (with $k=0.5 D$ ) ranged from 11.4 to 19.1 for the ' $\mathrm{M}$ ' series experiments, which are in the transitionally-rough regime between hydraulically-smooth $\left(R_{k^{*}}<4\right)$ and fully-rough $\left(R_{k^{*}}>60\right)$. Although the bed roughness was not measured for the ' $S$ ' series experiments, the painted bed surface is expected to be hydraulically-smooth with roughness Reynolds numbers much less than 1.

A 2-component scanning-beam Particle Image Velocimetry (PIV) system was used to measure fluid velocities at $100 \mathrm{~Hz}$ (Schlicke et al. 2007). The size of the measurement plane was approximately $38 \mathrm{~mm}$ wide (covering 4 marbles) and $80 \mathrm{~mm}$ high and orientated in the streamwise and bednormal directions. Measurements were conducted along the centreline of the flume, with the laser light sheet aligned so that it passed alternately through the crest of a sphere in one row, then midway between two spheres in the next row. In each experiment, the PIV recordings were made over a 4 minute period, with double-averaged quantities calculated over 24000 time steps and 27 streamwise positions. Images were analysed using rectangular interrogation windows, 32 pixels wide and 16 pixels high with a $50 \%$ overlap resulting in a vector spacing of $1.4 \mathrm{~mm}$ in the horizontal and $0.7 \mathrm{~mm}(<2.4$ wall units) in the vertical direction. The light sheet thickness, defining the extent of the sampling volume in the transverse direction, was $2 \mathrm{~mm}$. An iterative cross-correlation algorithm with a three-point Gaussian peak interpolation function was used to estimate velocity vectors. With each iteration, interrogation regions are offset and distorted according to the results of the previous analysis pass using a sin-cardinal image interpolation function (Scarano 2002). The iterative algorithm, although more computationally expensive than a single pass method, returns higher cross-correlation coefficients and reduces measurement noise.

\section{VELOCITY AND STRESS DISTRIBUTIONS}

In this section, we examine differences in double-averaged fluid velocities, turbulence intensities and stresses between hydraulically-smooth and transitionally-rough bed surfaces. We take the origin of the $z$ coordinate as the roughness tops for ' $\mathrm{M}$ ' series experiments, and as the level of the flat bed for ' $\mathrm{S}$ ' series experiments. The zero-plane displacement $d$ is defined here as $0.16 D$ (Bayazit 1983, see also Jackson 1981) for the spherical-roughness- 
element experiments and as zero for the smooth-bed experiments. Shear velocity is determined by extrapolation of the near-bed total fluid stress distribution to the roughness tops or the flat bed for the ' $M$ ' and ' $S$ ' series experiments, respectively. We use ' + ' notation to denote normalisation with inner flow variables $\left(u_{*}, v\right)$, i.e. $z^{+}=z u_{*} / v$ and $u^{+}=u / u *$.

Double-averaged streamwise velocities are plotted for all experiments in Fig. 1a. The smooth bed cases S1-3 follow the expected (dashed lines in Fig. 1a) linear distribution $\left\langle\overline{u^{+}}\right\rangle=z^{+}$within the viscous sublayer and the $\log$ law $\left\langle\overline{u^{+}}\right\rangle=\kappa^{-1} \ln \left(z^{+}+d^{+}\right)+C$, with $\kappa=0.38$ and $C=5.2$ for $z^{+}>40$ and $z^{+}<<H^{+}$. The transitional roughness data (M1-5) are also approximately logarithmic over a range of $z^{+}$and show the expected offset towards lower $\left\langle\overline{u^{+}}\right\rangle$with increasing roughness Reynolds number (e.g., Montes 1998). Velocity gradients for smooth- and transitionally-rough bed data (Fig. 1b) are found to collapse towards a common curve through to the top of the logarithmic layer $\left(z^{+}=60-100\right)$. Dashed lines in Fig. 1b indicate the expected velocity gradients in the viscous sublayer, $\mathrm{d}\left\langle\overline{u^{+}}\right\rangle / \mathrm{d} z^{+}=1$, and logarithmic regions, $\mathrm{d}\left\langle\overline{u^{+}}\right\rangle / \mathrm{d} z^{+}=\left[\kappa\left(z^{+}+d^{+}\right)\right]^{-1}$, which closely match the experimental data. Above the roughness tops, similarity of velocity-gradient, $\mathrm{d}\left\langle\overline{u^{+}}\right\rangle / \mathrm{d} z^{+}$, distributions implies equal contributions of viscous stress to the total fluid stress, $v \mathrm{~d}\langle\bar{u}\rangle / \mathrm{d} z / u_{*}^{2}$, for both smooth- and transitionally-rough beds. This similarity between transitionally-rough and smooth beds may suggest that wake eddies shed from roughness elements, which are feature of fully-rough bed flows, have little significance over the studied range of roughness Reynolds numbers. Below the roughness tops where form drag and skin friction act as a sink for momentum, an inflection point in the mean velocity profile and therefore a maximum in the velocity gradient distribution is expected (although our data is limited to the region above the roughness tops). Here,

(a)

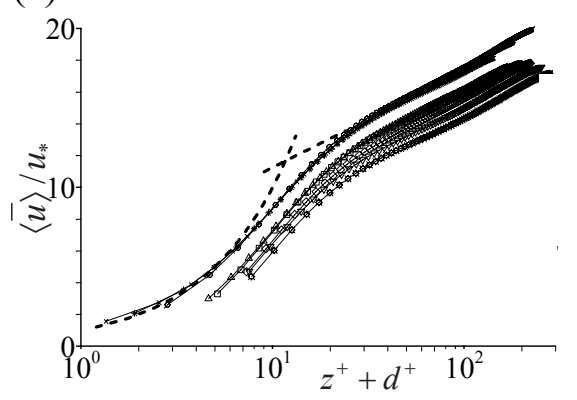

(b)

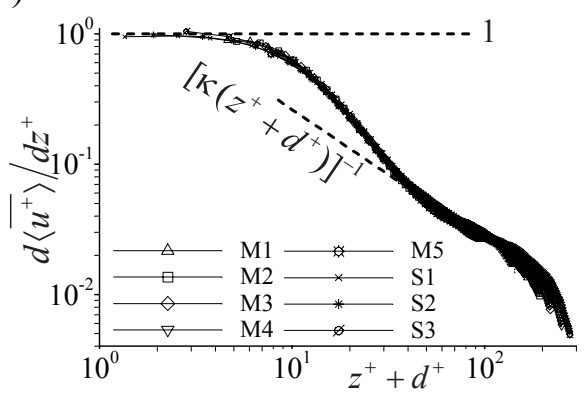

Fig. 1. Double-averaged velocity (a) and velocity-gradient (b) distributions. Roughness tops for M1-5 are in the range $z^{+}+d^{+}=3.7$ to 6.2 , and the bed level for S1-3 is at $z^{+}+d^{+}=0$. 
velocity gradients for the transitional-roughness experiments should diverge from the smooth-bed distributions.

Turbulence-intensity distributions are plotted in Fig. 2 for streamwise and bed-normal velocity components, with elevation normalised both by flow depth $H$ and the viscous length scale $\left(v / u_{*}\right)$. Away from the bed, $z / H>0.3$, where viscous effects become negligible, we find measured turbulence intensities for all experiments to approximately agree with values predicted from semi-empirical relationships (2) and (3), (Nezu and Nakagawa 1993), with $C_{1}=2.30$ and $C_{3}=1.27$.

$$
\begin{aligned}
& \left\langle\overline{\left.u^{\prime 2}\right\rangle^{0.5} / u_{*}}=C_{1} \exp (-z / H),\right. \\
& \left\langle\overline{w^{\prime 2}}\right\rangle^{0.5} / u_{*}=C_{3} \exp (-z / H) .
\end{aligned}
$$

For the smooth-bed experiments, streamwise turbulence intensity is found to be approximately linear within the viscous sublayer, consistent with the prediction of Levich (1962). In this range, we find a slope of approximately 0.34 (dashed line in Fig. 2b inset), equivalent to that identified by Lopez (1997) and similar to the value of 0.3 suggested by Nezu and Naka-
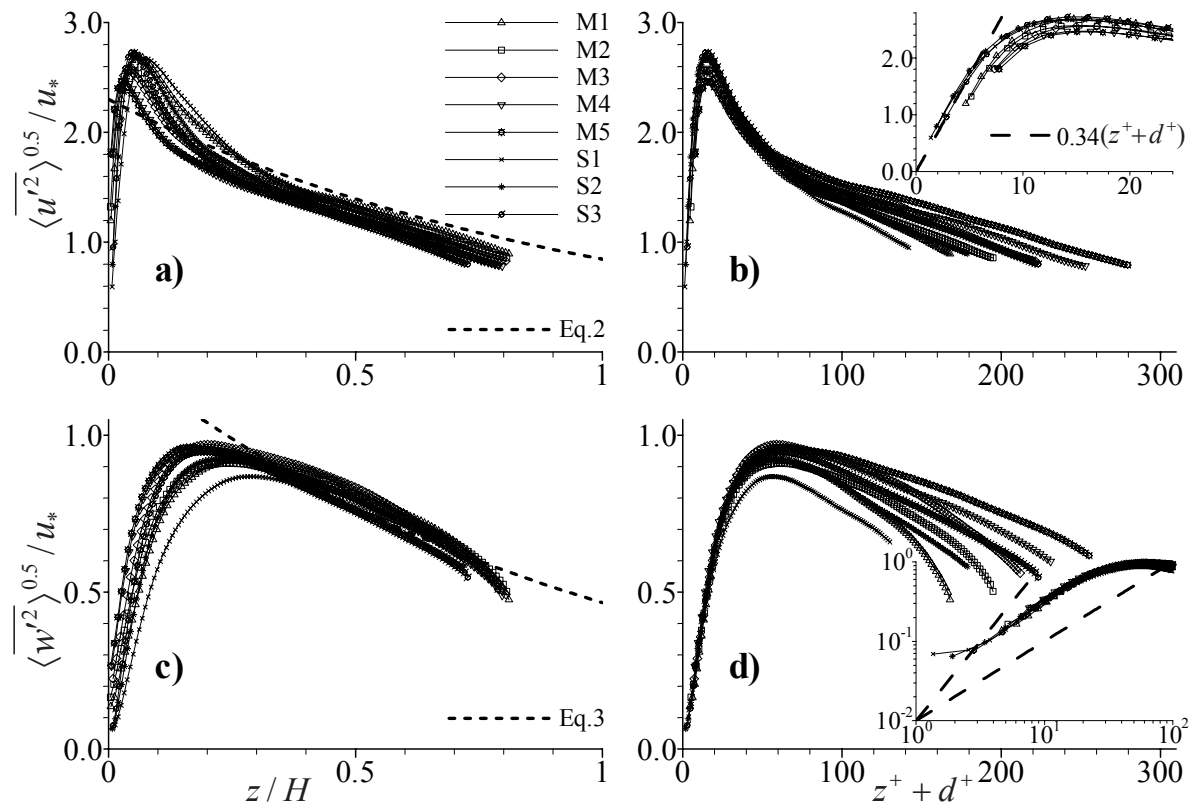

Fig. 2. Double-averaged turbulence intensity for streamwise (a) and (b), and vertical (c) and (d) velocities. Plots (a) and (c) are normalised with outer-flow variables, while (b) and (d) are normalised with inner variables. Insets in (b) and (d) are enlargements of the near bed region. Dashed lines in (d) are power laws with slopes 2 (upper) and 1 (lower) corresponding to parabolic and linear trends, respectively. 
gawa (1993). For the transitional roughness experiments, the peak turbulence intensity is found to decrease with increasing roughness Reynolds number, from $\left\langle\overline{u^{\prime 2}}\right\rangle^{0.5} / u_{*}=2.69$ at $R_{k^{*}}=11.4$, to $\left\langle\overline{\left\langle u^{\prime 2}\right.}\right\rangle^{0.5} / u_{*}=2.46$ at $R_{k^{*}}=19.1$. Nezu and Nakagawa (1993) show that this trend continues for increasing $R_{k^{*}}$ with peak turbulence intensity reaching a minimum of around 2.0 for fullyrough bed flows. This trend is attributed by Nezu and Nakagawa (1993) to large eddies being broken into smaller eddies by the roughness elements. The porous nature of the bed for tests M1-M5 may also act to absorb and dissipate turbulent energy. For all experiments, the elevation of the peak streamwise turbulence intensity remains approximately constant at $z^{+}+d^{+}=15$, similar to the values of $z^{+}=14$ identified by Moser et al. (1999) from direct numerical simulations and the $z^{+}=17$ suggested by Nezu and Nakagawa (1993) for flow over smooth boundaries. This suggests that relative to smooth beds, turbulent eddies for transitionally-rough beds are displaced downwards a distance $d$ into the fluid volume below the roughness tops (i.e., eddies are partially submerged into the bed). Since $d$ is the zero plane displacement for the logarithmic velocity distribution, and also appears to collapse the elevations of peak turbulence intensity, it may be possible to make use of turbulence intensity measurements when determining appropriate values of $d$ for transitionally-rough beds. Although eddy dynamics for fully-rough bed flows are likely to differ from their smooth and transitionally-rough bed counterparts, near-bed turbulence intensity distributions may still prove useful in evaluating $d$ for rough bed flows and should be studied further.

Vertical turbulence intensity peaks further away from the wall than streamwise turbulence intensity, consistent with the experimental measurements of Grass (1971) and Bigillon et al. (2006) and the DNS simulations of Spalart (1988) and Moser (1999). This effect may be due to the damping of wall-normal velocity fluctuations due to the presence of the bed, although surprisingly we see little difference here between the smooth (S1-S3) and porous beds (M1-M5). Few predictive curves are available for vertical turbulence intensity in the near-bed region. We tested Levich's (1962) prediction of a $\left\langle\overline{w^{\prime 2}}\right\rangle^{0.5} / u_{*} \propto z^{2}$ relation within the viscous sublayer, but our data appear closer to a linear relation than to a parabolic law (Fig. 2d).

Form-induced intensities (Fig. 3) are found to be maximum at the roughness tops for experiments M1-M5, and decay rapidly with increasing $z$ to become negligible at around $z / H=0.056$ for streamwise velocity and $z / H=0.076$ for vertical velocity. Since $H / D$ is approximately constant for all transitional-roughness experiments, we can translate these values to $z / D$ scaling and find $z / D=0.50$ and $z / D=0.68$ for streamwise and vertical form-induced intensities, respectively. These values identify the upper boun- 

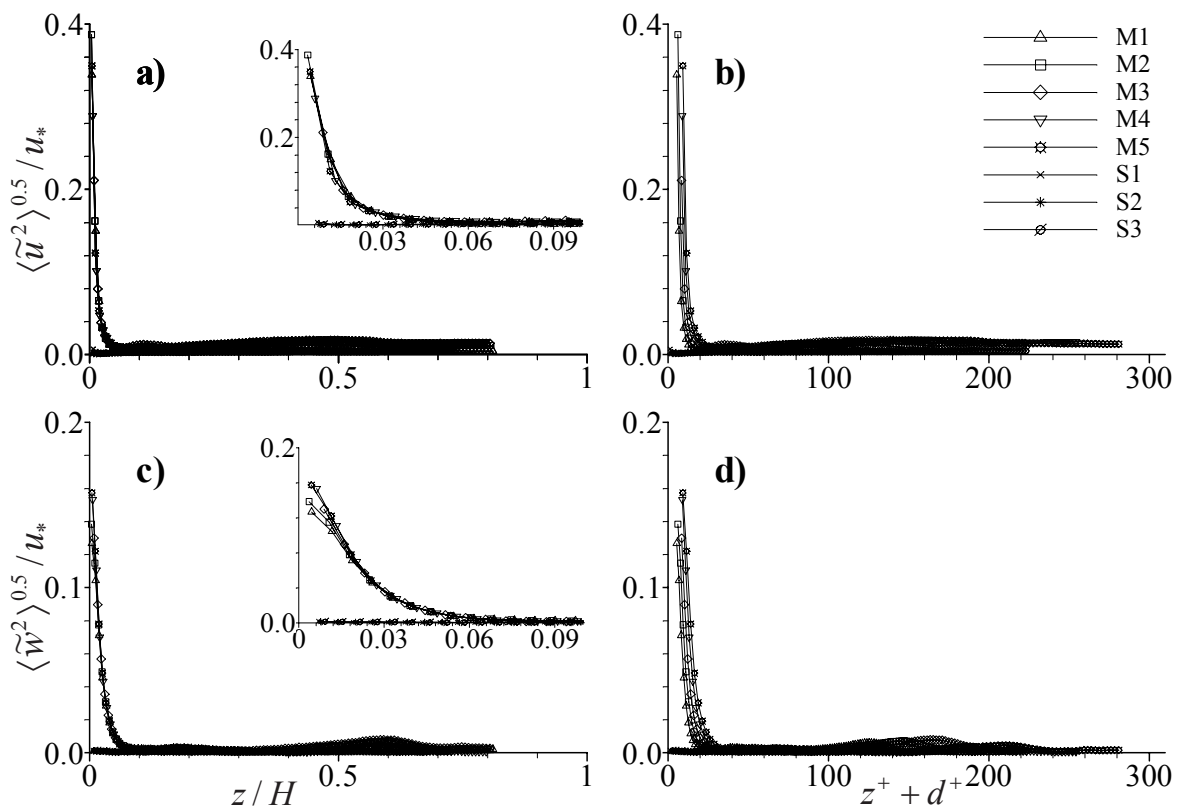

Fig. 3. Spatially-averaged form-induced intensity for streamwise (a) and (b), and vertical velocities (c) and (d). Plots (a) and (c) are normalised with outer-flow variables, while (b) and (d) are normalised with inner variables. Insets in (a) and (c) are enlargements of the near-bed region.

dary to the flow layer in which the mean flow is spatially heterogeneous, and are comparable to $z / D=0.6$ for flow over spheres in a cubic packing $\left(R_{k^{*}}=137-216\right)$ identified by Manes et al. (2007). Peak magnitudes of $\left\langle\widetilde{u}^{2}\right\rangle^{0.5} / u_{*}$ and $\left\langle\widetilde{w}^{2}\right\rangle^{0.5} / u_{*}$ for the measured data are around 0.40 and 0.16 , respectively, markedly smaller than the values of 3.47 and 0.22 identified by Manes et al. (2007). This discrepancy may be due either to the different packing arrangements adopted for the spheres (cubic for Manes et al. 2007, and hexagonal in the present study), or to the different ranges of roughness Reynolds numbers studied (137-216 for Manes et al. 2007, and 11.4-19.1 in the present study). It is interesting to note that there is no noticeable Reynolds number dependence in our measured data, with all points collapsing towards a common curve (with $z / H$ normalisation) for both streamwise and vertical form-induced intensities. With elevation normalised by the viscous length scale (Figs. 3b,d), measured data points fall on separate curves. Although the applicability of the zero plane displacement for logarithmic velocity distributions $d$ to form induced intensity distributions is questionable, we use it in Fig. 3 to maintain consistency with the other plots presented.

Viscous, Reynolds, form-induced and total stress distributions for all measured data are plotted in Fig. 4. The total stress distribution is found to 

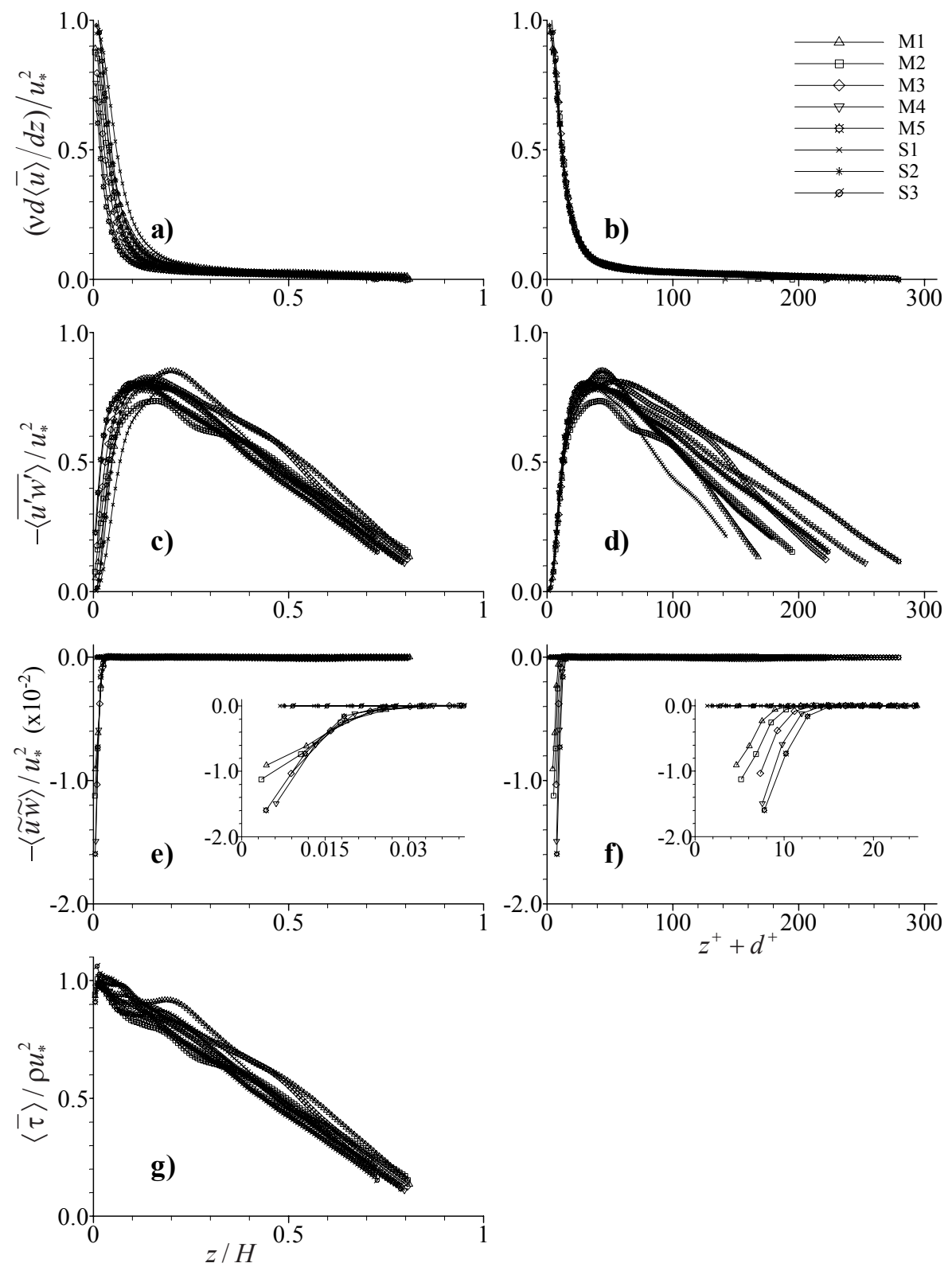

Fig. 4: Viscous (a) and (b), Reynolds (c) and (d), form-induced (e) and (f), and total (g) fluid stresses. Left column elevation scale is normalised with flow depth, right column elevation is normalised with inner-flow variables. Insets in (e) and (f) are enlargements of near-bed region. 
approximate the expected linear trend between the water surface and the roughness tops. This suggests that the studied flows are nearly twodimensional, with minimal contribution of secondary currents to the total momentum flux. At the level of the roughness tops for experiments M1-M5, and at the smooth-bed level for S1-S3, the viscous stresses make the dominant contribution to the total stress. The increase in viscous stresses approaching the boundary is balanced by a reduction in Reynolds stresses, which fall towards zero at the bed level. Form-induced stresses are small with a peak magnitude of around $1.5 \%$ of the total stress, but they are of opposite sign to Reynolds and viscous stresses. This contrasts with the study of Manes et al. (2007) where form induced stresses were found to be positive and larger in magnitude ( $25 \%$ of total stress at the roughness tops), potentially due to the different sphere packing arrangement and roughness Reynolds numbers studied. As with form-induced intensity, we note that forminduced shear stresses appear to scale independent of viscous effects, with data collapsing towards a common curve when the elevation scale is normalised by flow depth. Moving away from the bed, form-induced shear stresses quickly decay and reach zero at around $z / H=0.03$ or $z / D=0.27$; quite similar to the $z / D=0.16-0.3$ range seen in the data of Manes et al. (2007).

\section{DISCUSSION AND CONCLUSIONS}

We use the framework of the double-averaged Navier-Stokes equations to characterise velocity and stress distributions over smooth and transitionallyrough beds. Experimental measurements were made using PIV, with bed roughness created using $11 \mathrm{~mm}$ diameter spheres in a hexagonal packing. Oil with a viscosity 15 times that of water was used to reduce roughness Reynolds numbers to the range $R_{k^{*}}=11.4-19.1$.

Results indicate that form-induced intensities (the square root of forminduced normal stress) decay rapidly from a maximum at the roughness tops to vanish at $z / D=0.50$ and $z / D=0.68$ for streamwise and bed-normal velocities respectively. Form-induced shear stresses were found to be negative and vanish at a distance of $z / D=0.27$ from the roughness tops. Magnitudes of bed-normal form-induced intensity are comparable to those identified by Manes et al. (2007) for cubic sphere packing and $R_{k^{*}}=137-216$, but streamwise form-induced intensities and form-induced shear stresses are an order of magnitude smaller. The relative significance of the bed packing arrangement and the roughness Reynolds numbers for form-induced stresses remains to be clarified, but our data do not reveal any Reynolds number dependence over the range of $R_{k^{*}}=11.4-19.1$.

A linear relationship between streamwise turbulence intensity and $z^{+}$ with slope 0.34 is confirmed within the viscous sublayer for smooth-bed 
flows. Streamwise turbulence intensities are found to reduce in magnitude with increasing $R_{k^{*}}$, consistent with Nezu and Nakagawa (1993), but the elevation of maximum turbulence intensity remains constant at $z^{+}+d^{+}=15$, where $d$ is the zero plane displacement for the logarithmic velocity profile. This suggests that turbulence intensity distributions may be used in assessing the origin of logarithmic velocity profiles for transitionally-rough bed flows by measuring the relative shift in the elevation of peak turbulence intensity compared to that for smooth boundaries. A similar approach may also be applicable to fully-rough boundaries and should be studied further. Wallnormal turbulence intensities do not reveal any dependence on roughness Reynolds number.

Measured double-averaged velocity distributions for smooth beds closely follow predicted curves in the viscous sublayer and logarithmic regions. Equivalent distributions for transitionally-rough beds become increasingly offset from smooth-bed profiles with increasing $R_{k^{*}}$, but surprisingly velocity- gradient distributions above the roughness tops do not show any dependence on roughness. We interpret this as an indication of limited wake eddy shedding for these low roughness Reynolds number flows.

Acknowledgements. The authors are grateful for the comments and suggestions from the two anonymous reviewers.

\section{References}

Bayazit, M. (1983), Flow structure and sediment transport mechanics in steep channels. In: B.M. Sumer and A. Müller (eds.), Euromech 156: Mechanics of Sediment Transport, Balkema, Instanbul, 197-206.

Bigillon, F., Y. Niño, and M.H. Garcia (2006), Measurements of turbulence characteristics in an open-channel flow over a transitionally-rough bed using particle image velocimetry, Experiments in Fluids 41, 6, 857-867.

Grass, A.J. (1971), Structural features of turbulent flow over smooth and rough boundaries, J. Fluid Mechanics 50, 2, 233-255.

Jackson, P.S. (1981), On the displacement height in the logarithmic velocity profile, J. Fluid Mechanics 111, 15-25.

Krogstad, P.-A., R.A. Antonia, and L.W.B. Browne (1992), Comparison between rough- and smooth-wall turbulent boundary layers, J. Fluid Mechanics 245, 599-617.

Levich, V.G. (1962), Physicochemical Hydrodynamics, Prentice-Hall, New Jersey.

Ligrani, P.M., and R.J. Moffat (1986), Structure of transitionally rough and fully rough turbulent boundary layers, J. Fluid Mechanics 162, 69-98. 
Lopez, F. (1997), Open-channel flow with roughness elements of different spanwise aspect ratios: turbulence structure and numerical modeling, $\mathrm{PhD}$ thesis, University of Illinois, Urbana-Champaign, IL.

Manes, C., D. Pokrajac, and I. McEwan (2007), Double-averaged open-channel flows with small relative submergence, J. Hydraul. Eng. 133, 8, 896-904.

Monin, A.S., and A.M. Yaglom (1971), Statistical Fluid Mechanics: Mechanics of Turbulence, Vol. 1, MIT Press, Boston.

Montes, J.S. (1998), Hydraulics of Open Channel Flow, ASCE Press, New York.

Moser, R.D., J. Kim, and N.N. Mansour (1999), Direct numerical simulation of turbulent channel flow up to $\mathrm{Re}_{\tau}=590$, Physics of Fluids 11, 4, 943-945.

Nezu, I., and H. Nakagawa (1993), Turbulence in Open-Channel Flows, Balkema, Rotterdam.

Nikora, V., I. McEwan, S. McLean, S. Coleman, D. Pokrajac, and R. Walters (2007a), Double-averaging concept for rough-bed open-channel and overland flows: Theoretical background, J. Hydraul. Eng. 133, 8, 873-883.

Nikora, V., S. McLean, S. Coleman, D. Pokrajac, I. McEwan, L. Campbell, J. Aberle, D. Clunie, and K. Koll (2007b), Double-averaging concept for rough-bed open-channel and overland flows: Applications, J. Hydraul. Eng. 133, 8, 884-895.

Scarano, F. (2002), Iterative image deformation methods in PIV, Measurement Science and Technology 13, 1, R1-R19.

Schlicke, T., S.M. Cameron, and S.E. Coleman (2007), Galvanometer-based PIV for liquid flows, Flow Measurement and Instrumentation, 18, 1, 27-36.

Schultz, M.P., and K.A. Flack (2007), The rough-wall turbulent boundary layer from the hydraulically smooth to the fully rough regime, J. Fluid Mechanics 580, 381-405.

Spalart, P.R. (1988), Direct simulation of a turbulent boundary layer up to $R_{\theta}=1410$, J. Fluid Mechanics 187, 61-98. 